\title{
Immature Monocyte to Leukocyte Ratio Measurement
}

National Cancer Institute

\section{Source}

National Cancer Institute. Immature Monocyte to Leukocyte Ratio Measurement. NCI

Thesaurus. Code C96677.

The determination of the ratio of immature monocytes compared to total leukocytes present in a sample. The measurement may be expressed as a ratio or percentage. 